\title{
Non-achievement of the Low-Density Lipoprotein Cholesterol Goal in Older Patients with Type 2 Diabetes Mellitus and a Very High Cardiovascular Disease Risk: A Multicenter Study in Vietnam
}

\author{
Huan Thanh Nguyen ${ }^{1,2}$, Khang Pham Trong $\mathrm{Ha}^{2,3}$, An Huu Nguyen ${ }^{1}$, Thu Thanh Nguyen ${ }^{1}$, Hang My Lam ${ }^{1}$ \\ ${ }^{1}$ Department of Geriatrics and Gerontology, University of Medicine and Pharmacy at Ho Chi Minh City, Ho Chi Minh, Vietnam \\ ${ }^{2}$ Department of Cardiology, Thong Nhat Hospital, Ho Chi Minh, Vietnam \\ ${ }^{3}$ Department of Geriatrics and Gerontology, Pham Ngoc Thach University of Medicine, Ho Chi Minh, Vietnam
}

Corresponding Author:

Huan Thanh Nguyen, $\mathrm{MD}, \mathrm{PhD}$

Department of Geriatrics and

Gerontology, University of Medicine

and Pharmacy at Ho Chi Minh City,

217 Hong Bang Street, Ward 11,

District 5, Ho Chi Minh City, Vietnam

E-mail: huannguyen@ump.edu.vn

ORCID:

https://orcid.org/0000-0002-5086-7273

Received: September 3, 2021

Revised: November 2, 2021

Accepted: November 3, 2021
Background: Lowering the low-density lipoprotein cholesterol (LDL-c) level is important for reducing cardiovascular (CV) events. However, little is known about the management of LDL-c in older patients with type 2 diabetes mellitus (T2DM). This study investigated the prevalence and factors associated with the non-achievement of LDL-c goals in older T2DM patients with a very high risk of CV diseases. Methods: This multicenter cross-sectional study measured the LDL-C levels of 733 T2DM outpatients from December 2019 to July 2020. The patients were aged $\geq 60$ years, had very high risks of CV disease, and had been on LDL-c-lowering therapy for 6 months or more. The goal of lipid concentrations were assessed based on the recent guidelines of the European Society of Cardiology. We applied logistic regression analysis to identify the factors associated with the non-achievement of the LDL-c goal. Results: The mean age of the patients was $68.6 \pm 7.2$ years. In total, 654 patients (89.2\%) did not achieve the aggressive LDL-c goal of $<1.4$ $\mathrm{mmol} / \mathrm{L}$. In the adjusted model, the factors associated with the non-achievement of the LDL-c goal were obesity defined by body mass index (odds ratio $[\mathrm{OR}]=2.33 ; 95 \%$ confidence interval $[\mathrm{Cl}, 1.13-4.81 ; \mathrm{p}=0.022)$ and high-intensity statin therapy $(\mathrm{OR}=0.03 ; 95 \% \mathrm{Cl}, 0.01-0.05$; $\mathrm{p}<0.001)$, while age, sex, education level, smoking habit, and comorbidities were not associated. Conclusion: Older patients with T2DM who are at a very high CV disease risk are often unable to achieve their LDL-c goal. Obesity can increase the probability of not achieving the LDL-c goal, whereas high-intensity statin therapy can decrease this probability.

Key Words: Low-density lipoprotein (LDL) cholesterol, Diabetes mellitus, Older patients, Aged

\section{INTRODUCTION}

Type 2 diabetes mellitus (T2DM) is a major global health burden in currently aging societies. ${ }^{1)}$ Diabetes management in older adults is difficult owing to the coexistence of geriatric syndromes such as frailty, falling, delirium, polypharmacy, and polymorbidity. ${ }^{2}$ Vietnam has entered an aging phase: in 2019, people aged $\geq 60$ years accounted for $12.3 \%$ of the total Vietnamese population. ${ }^{3)}$ Furthermore, the country has been experiencing an epidemiological transition, with the health panorama shifting from infectious to non-communicable diseases. ${ }^{4)}$ Previous studies showed that $6 \%$ of the total population in Vietnam had diabetes, ${ }^{5)}$ with the rate increasing to $12.5 \%$ among older individuals. ${ }^{6}$ Dyslipidemia was detected in $37.9 \%-60.2 \%$ of older adults with T2DM. ${ }^{7,8)}$ Several factors reflecting risk-behavior changes drive these high rates, including smoking, unhealthy eating habits, and a sedentary lifestyle. ${ }^{9)}$

Until recently, atherosclerotic cardiovascular disease (ASCVD) was the principal cause of morbidity and mortality in patients with diabetes. ${ }^{10,11)}$ Previous studies demonstrated that lowering the low-density lipoprotein cholesterol (LDL-c) level prevented and 
reduced the rate of ASCVD events in patients with T2DM. ${ }^{12-15)}$ The relative reduction in the rate of ASCVD events was also observed in older patients with T2DM. ${ }^{15)}$ Notably, the approach to achieving lipid goals is an important part of the comprehensive ASCVD risk reduction strategy. In a cohort study of 69,942 patients in South Korea, Kim et al. ${ }^{16)}$ reported a higher rate of ASCVD events among those who did not achieve the LDL-c goal compared to those who did. The LDL-c goal non-achievers were older and had higher cardiovascular (CV) risk levels compared to the LDL-c goal achievers.

The recent clinical guidelines of the European Society of Cardiology/European Atherosclerosis Society (ESC/EAS) for dyslipidemia and the European Society of Cardiology/European Association for the Study of Diabetes (ESC/EASD) for diabetes recommend an LDL-c goal of $<1.4 \mathrm{mmol} / \mathrm{L}$ for patients with T2DM who are at very high risk for $\mathrm{CV}$ disease. ${ }^{17,18)}$ However, there is a lack of evidence of non-achievement of LDL-c goals in older patients with T2DM at very high risk for CV disease. Therefore, this cross-sectional study aimed to determine how often LDL-c goals are not achieved in very high-risk older patients with T2DM and identify the factors associated with non-achievement.

\section{MATERIALS AND METHODS}

\section{Sample Size Calculation}

The sample size was calculated using a single population proportion formula:

$$
N=Z_{1-\alpha / 2}^{2} \times\left(p \times(1-p) / d^{2}\right),
$$

where $N$ is the required sample size; $Z_{1-\alpha / 2}$ is 1.96 (with $\alpha=0.05$ and $95 \%$ confidence interval $[\mathrm{CI}]$ ); $d$ is precision (assumed to be $0.04)$; and $p$ is the prevalence of not achieving the LDL-c goal $(<1.4 \mathrm{mmol} / \mathrm{L})$ in older T2DM patients at a very high $\mathrm{CV}$ disease risk. Because the prevalence was unknown, we set $p=0.5$ to obtain the maximum possible value of $p \times(1-p)$ of 0.25 . This study required a minimum of 600 participants.

\section{Ethical Statement}

The study was carried out according to the ethical principles of the Declaration of Helsinki. All patients provided written informed consent. The study protocol was approved by the ethics committee of the University of Medicine and Pharmacy at Ho Chi Minh City, Vietnam (No. 2545/QD-DHYD).

\section{Study Design and Data Collection}

This multicenter cross-sectional study was conducted from De- cember 2019 to July 2020 in outpatients with T2DM aged $\geq 60$ years at three hospitals in Ho Chi Minh City, Vietnam. To ensure consistent management among the study sites, trained geriatricians treated all patients according to the ESC guidelines ${ }^{17,18)}$ and all sites started recruitment at the same time. Patients met the inclusion criteria if they had been treated for hypercholesterolemia with LDL-c-lowering therapy for 6 or more months before enrollment. The CV risk category and lipid goals were assessed according to the recent ESC guidelines. ${ }^{17,18)}$

Patients were diagnosed with diabetes based on a fasting plasma glucose level of $\geq 7.0 \mathrm{mmol} / \mathrm{L}$ after no caloric intake for at least 8 hours and/or an HbAlc level of $\geq 6.5 \% .{ }^{18)}$ Hypercholesterolemia was diagnosed in our very-high-risk patients if their LDL-c levels were $>1.4 \mathrm{mmol} / \mathrm{L}$ before lipid management. ${ }^{17)}$ In our study, all T2DM patients with hypercholesterolemia and at least two major risk factors (age $\geq 65$ years, hypertension, smoking, and obesity) were classified as having a very high $\mathrm{CV}$ disease risk. ${ }^{17)}$ For participants with very high CV disease risk, the lipid goal was an LDL-c level of $<1.4 \mathrm{mmol} / \mathrm{L}$, a non-high-density lipoprotein cholesterol (non-HDL-c) level of $<2.2 \mathrm{mmol} / \mathrm{L}$, and a triglyceride level of $<1.7 \mathrm{mmol} / \mathrm{L} .{ }^{17)}$ All blood samples for serum lipid estimation were obtained after a 12-hour fast. LDL-c levels were measured using a direct LDL-c assay kit (LDL-CHOLESTEROL, OSR6183; Beckman Coulter Ireland Inc., Clare, Ireland). The exclusion criteria were severe illnesses requiring hospital admission, serious mental conditions, and active malignancies.

The geriatricians managing the participating patients collected participant demographic data and clinical characteristics and measured their body weights and heights. The intensity of statin therapy was defined based on the last prescription within one month before enrollment. High-intensity statin therapy included 40-80 $\mathrm{mg}$ atorvastatin or $20-40 \mathrm{mg}$ rosuvastatin, whereas low-to-moderate intensity statin therapy was defined based on lower doses of all statins. ${ }^{17)}$ The participants' educational level was classified as lower ( $\leq 12$ years of education, including graduation from senior high school or lower) or higher (graduation from college or university or higher). Patients who had smoked any tobacco products over the previous year were considered current smokers. Body mass index (BMI) was calculated as the quotient between body weight $(\mathrm{kg})$ and height $\left(\mathrm{m}^{2}\right)$. Body weight and height were measured following the standardized protocol using identical equipment at all study sites. BMI was stratified per the World Health Organization's guidelines for the Asia-Pacific region, which defined underweight $\left(<18.5 \mathrm{~kg} / \mathrm{m}^{2}\right)$, normal weight $\left(18.5-22.9 \mathrm{~kg} / \mathrm{m}^{2}\right)$, overweight $\left(23.0-24.9 \mathrm{~kg} / \mathrm{m}^{2}\right)$, and obese $\left(\geq 25 \mathrm{~kg} / \mathrm{m}^{2}\right) .{ }^{19)}$ The normal weight group was the reference group for regression analyses. 


\section{Statistical Analyses}

All data were analyzed using IBM SPSS Statistics for Windows, version 25.0 (IBM Corp., Armonk, NY, USA). Qualitative data were described as frequencies and percentages. Quantitative data were represented as mean \pm standard deviation. Comparisons were conducted with chi-square or Fisher exact tests for non-numerical data and Student t-test for continuous variables. Univariate and multivariate logistic regression analyses using the enter method were performed to identify the predictors of non-achievement. Univariate logistic regression was performed on the potential risk factors for non-achievement. All variables were selected for multivariate logistic regression and examined for interaction and multicollinearity. The significance level was set at $\mathrm{p}<0.05$.

\section{RESULTS}

\section{Patient Characteristics and the Prevalence of Not Achieving the Lipid Goal}

We enrolled 733 patients with a mean age of $68.6 \pm 7.2$ years (range, 60-94 years). The participants were predominantly male (51.3\%). Overall, 71 patients (9.7\%) were current smokers, 174 (23.7\%) had completed college/university or higher education, $412(56.2 \%)$ had documented coronary artery disease (CAD), and 575 patients reported hypertension (78.4\%). Regarding BMI, $2.3 \%$ were underweight, $34.7 \%$ were of normal weight, $26.6 \%$ were overweight, and $36.4 \%$ were obese.

A total of 654 patients $(89.2 \%)$ did not achieve the aggressive LDL-c goal $(<1.4 \mathrm{mmol} / \mathrm{L})$. Further, 644 (87.9\%) did not achieve the non-HDL-c goal $(<2.2 \mathrm{mmol} / \mathrm{L}), 412(56.2 \%)$ did not meet the triglyceride goal $(<1.7 \mathrm{mmol} / \mathrm{L})$, and $355(48.4 \%)$ did not meet the $\mathrm{HbAlc}$ goal $(<7 \%)$, indicating a high prevalence of non-achievement in older patients with T2DM with a very high $\mathrm{CV}$ disease risk.

\section{Differences between the Groups That Did and Did Not Achieve the LDL-c Goal}

The participants were assigned to achieved or non-achieved LDL-c goal groups. Table 1 presents the baseline characteristics of all participants and compares the two groups. Age, sex, education levels, hypertension, and CAD did not differ between the groups. As expected, the non-achieved group had a significantly higher LDL-c level than that in the achieved group ( $2.9 \pm 1.0$ vs. $1.1 \pm 0.2$ $\mathrm{mmol} / \mathrm{L} ; \mathrm{p}<0.001$ ), along with a higher non-HDL-c level $(3.8 \pm 1.2$ vs. $2.2 \pm 0.9 \mathrm{mmol} / \mathrm{L} ; \mathrm{p}<0.001)$, a lower achievement rate for the non-HDL-c goal (5.4\% vs. $68.4 \%$; $\mathrm{p}<0.001$ ), and a higher BMI $\left(24.1 \pm 3.3\right.$ vs. $\left.22.9 \pm 3.4 \mathrm{~kg} / \mathrm{m}^{2} ; \mathrm{p}=0.003\right)$.

The distribution of patients according to BMI also differed sig- nificantly between the two groups ( $p=0.008$ ), with patients in the non-achieved group more likely to be obese compared to the achieved group (38.2\% vs. $21.5 \%$ ) (Fig. 1). Thus, obesity occurred more frequently in older patients with T2DM with a very high CV disease risk who did not achieve their LDL-c goal compared to those who did achieve the goal. The non-achieved group also had a significantly lower rate of high-intensity statin therapy than the achieved group ( $14.7 \%$ vs. $86.1 \%$; $<<0.001)$. We observed no significant difference in ezetimibe use between the two achievement groups (Supplementary Tables S1-S3).

\section{Factors Associated with Not Achieving the LDL-c Goal}

The results of the univariate and multivariate logistic regression analyses are summarized in Table 2 . In the adjusted model, obesity defined by BMI (odds ratio $[\mathrm{OR}]=2.33$; 95\% CI, 1.13-4.81; $\mathrm{p}=0.022)$ increased the probability of not achieving the LDL-c goal, whereas the adjusted ORs were 0.78 (95\% CI, 0.16-3.94; $\mathrm{p}=0.775$ ) for underweight and 1.33 (95\% CI, 0.66-2.68; $\mathrm{p}=0.423$ ) for overweight participants (normal weight was the reference). High-intensity statin use ( $\mathrm{OR}=0.03 ; 95 \% \mathrm{CI}, 0.01-0.05$; $\mathrm{p}<0.001)$ decreased the probability of not achieving the LDL-c goal.

\section{DISCUSSION}

The results of this multicenter cross-sectional study provided three key observations regarding the management of hypercholesterolemia in older patients with T2DM with a very high CV disease risk. First, a large proportion of patients did not meet the aggressive LDL-c goal. Second, obesity and the suboptimal use of high-intensity statin therapy were associated with not achieving the LDL-C goal. Third, those participants who did not meet the LDL-c goal also had lower achievement rates for the other goals, such as non-HDL-c, compared to those who achieved the LDL-c goal. According to these findings, we propose three discussion points.

\section{Non-achievement of the LDL-c Goal in Older Patients with T2DM at Very High CV Disease Risk}

Our study is the first to assess the achievement of an aggressive LDL-c target value (according to the 2019 ESC/EAS guidelines) in older patients with T2DM with a very high risk of CV disease. We found that $89.2 \%$ of patients did not achieve the recommended LDL-c goal of $<1.4 \mathrm{mmol} / \mathrm{L}$, despite being at a very high risk of having an ASCVD event. For the more relaxed LDL-c goals of $<1.8 \mathrm{mmol} / \mathrm{L}$ and $<2.6 \mathrm{mmol} / \mathrm{L}$, the non-achievement rates were $78.0 \%$ and $49.9 \%$, respectively (Fig. 1), corroborating the 
Table 1. Baseline characteristics of the patients according to the achievement of the goal LDL-c level

\begin{tabular}{|c|c|c|c|c|}
\hline Characteristic & All $(\mathrm{n}=733)$ & Achieved group $(\mathrm{n}=79)$ & Non-achieved group $(\mathrm{n}=654)$ & p-value ${ }^{a}$ \\
\hline Sex, male & $376(51.3)$ & $37(46.8)$ & $339(51.8)$ & 0.471 \\
\hline Age (y) & $68.6 \pm 7.2$ & $69.4 \pm 7.1$ & $68.5 \pm 7.2$ & 0.260 \\
\hline$\geq 75$ & $153(20.9)$ & $18(22.8)$ & $135(20.6)$ & 0.767 \\
\hline Education & & & & 0.440 \\
\hline Lower education & $559(76.3)$ & $57(72.2)$ & $502(76.8)$ & \\
\hline Higher education & $174(23.7)$ & $22(27.8)$ & $152(23.2)$ & \\
\hline $\mathrm{BMI}\left(\mathrm{kg} / \mathrm{m}^{2}\right)$ & $23.9 \pm 3.3$ & $22.9 \pm 3.4$ & $24.1 \pm 3.3$ & 0.003 \\
\hline BMI groups & & & & 0.008 \\
\hline Underweight & $17(2.3)$ & $3(3.8)$ & $14(2.1)$ & \\
\hline Normal & $254(34.7)$ & $39(49.4)$ & $215(32.9)$ & \\
\hline Overweight & $195(26.6)$ & $20(25.3)$ & $175(26.8)$ & \\
\hline Obese & $267(36.4)$ & $17(21.5)$ & $250(38.2)$ & \\
\hline Smoking & $71(9.7)$ & $6(7.6)$ & $65(9.9)$ & 0.640 \\
\hline Hypertension & $575(78.4)$ & $64(81.0)$ & $511(78.1)$ & 0.658 \\
\hline Coronary artery disease & $412(56.2)$ & $48(60.8)$ & $364(55.7)$ & 0.457 \\
\hline \multicolumn{5}{|l|}{ Lipid profile } \\
\hline Total cholesterol $(\mathrm{mmol} / \mathrm{L})$ & $4.8 \pm 1.4$ & $3.3 \pm 0.9$ & $5.0 \pm 1.3$ & $<0.001$ \\
\hline $\mathrm{LDL}-\mathrm{c}(\mathrm{mmol} / \mathrm{L})$ & $2.7 \pm 1.1$ & $1.1 \pm 0.2$ & $2.9 \pm 1.0$ & $<0.001$ \\
\hline HDL-c (mmol/L) & $1.1 \pm 0.3$ & $1.1 \pm 0.4$ & $1.1 \pm 0.3$ & 0.170 \\
\hline Non-HDL-c (mmol/L) & $3.7 \pm 1.2$ & $2.2 \pm 0.9$ & $3.8 \pm 1.2$ & $<0.001$ \\
\hline Triglyceride $(\mathrm{mmol} / \mathrm{L})$ & $2.2 \pm 1.5$ & $2.4 \pm 1.9$ & $2.2 \pm 1.5$ & 0.310 \\
\hline Non-HDL-c $\geq 2.2 \mathrm{mmol} / \mathrm{L}$ & $644(87.9)$ & $25(31.6)$ & $619(94.6)$ & $<0.001$ \\
\hline Triglyceride $\geq 1.7 \mathrm{mmol} / \mathrm{L}$ & $412(56.2)$ & $49(62.0)$ & $363(55.5)$ & 0.330 \\
\hline \multicolumn{5}{|l|}{ Diabetes profile } \\
\hline $\mathrm{HbAlc}(\%)$ & $7.4 \pm 1.8$ & $7.3 \pm 1.6$ & $7.4 \pm 1.8$ & 0.700 \\
\hline $\mathrm{HbAlc} \geq 7 \%$ & $355(48.4)$ & $33(41.8)$ & $322(49.2)$ & 0.257 \\
\hline \multicolumn{5}{|l|}{ LDL-c-lowering therapy } \\
\hline High-intensity statin therapy & $164(22.4)$ & $68(86.1)$ & $96(14.7)$ & $<0.001$ \\
\hline Ezetimibe & $27(3.7)$ & $2(2.5)$ & $25(3.8)$ & 0.758 \\
\hline
\end{tabular}

Values are presented as number (\%) or mean \pm standard deviation.

BMI, body mass index; HbAlc, glycated hemoglobin; HDL-c, high-density lipoprotein cholesterol; LDL-c, low-density lipoprotein cholesterol. Comparisons were conducted using chi-square and Student t-tests for categorical and continuous variables, respectively.

a) Achieved group vs. non-achieved group.

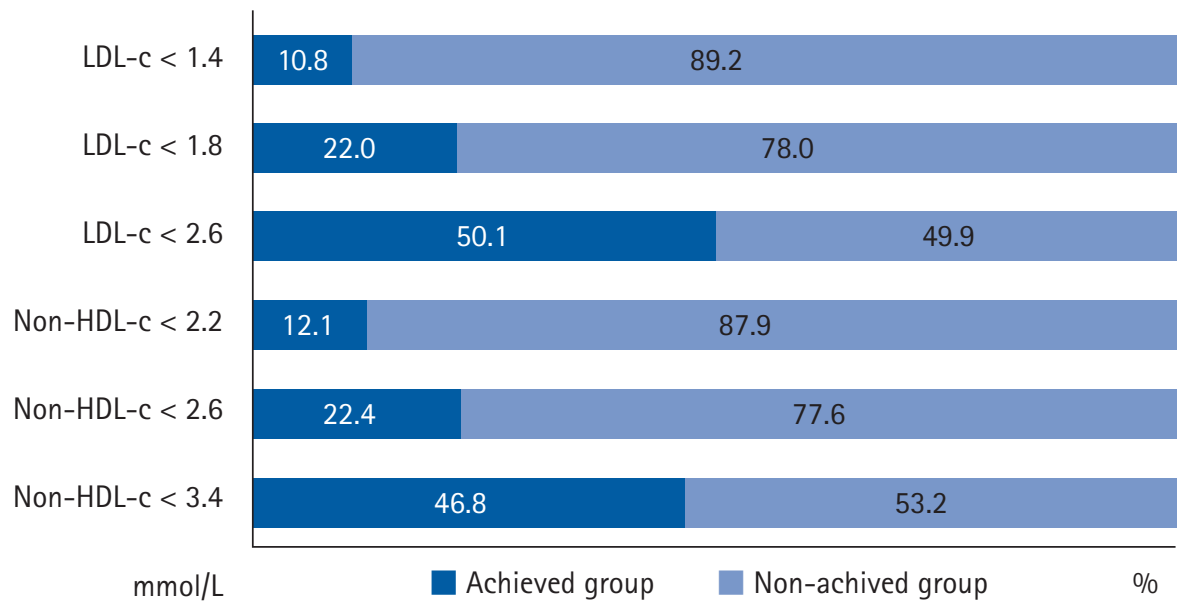

Fig. 1. Prevalence of the achievement and non-achievement of low-density lipoprotein cholesterol (LDL-c) and non-high-density lipoprotein cholesterol (non-HDL-c) goals according to the levels of treatment goals. 
Table 2. Factors associated with the non-achievement of LDL-c goal levels in logistic regression analysis

\begin{tabular}{|c|c|c|c|c|}
\hline \multirow{2}{*}{ Variable } & \multicolumn{2}{|c|}{ Univariate } & \multicolumn{2}{|c|}{ Multivariate } \\
\hline & OR $(95 \% \mathrm{CI})$ & p-value & $\operatorname{AOR}(95 \% \mathrm{CI})$ & $\mathrm{p}$-value \\
\hline Age & $0.98(0.95-1.01)$ & 0.262 & - & - \\
\hline Male & $1.22(0.77-1.95)$ & 0.402 & - & - \\
\hline Lower education & $1.28(0.76-2.15)$ & 0.364 & - & - \\
\hline \multicolumn{5}{|l|}{ BMI groups } \\
\hline Normal & 1 (Ref) & & 1 (Ref) & \\
\hline Underweight & $0.85(0.23-3.08)$ & 0.801 & $0.78(0.16-3.94)$ & 0.775 \\
\hline Overweight & $1.59(0.89-2.82)$ & 0.115 & $1.33(0.66-2.68)$ & 0.423 \\
\hline Obese & $2.67(1.47-4.85)$ & 0.001 & $2.33(1.13-4.81)$ & 0.022 \\
\hline High-intensity statin therapy & $0.03(0.01-0.06)$ & $<0.001$ & $0.03(0.01-0.05)$ & $<0.001$ \\
\hline Smoking & $1.34(0.56-3.21)$ & 0.507 & - & - \\
\hline Hypertension & $0.84(0.46-1.51)$ & 0.557 & - & - \\
\hline Coronary artery disease & $0.81(0.50-1.30)$ & 0.389 & - & - \\
\hline
\end{tabular}

LDL-c, low-density lipoprotein cholesterol; BMI, body mass index; CI, confidence interval; OR, odds ratio; AOR, adjusted odds ratio.

findings reported in other diabetic populations. Two recent studies in France and Korea showed that 59\% of patients with diabetes with very high $\mathrm{CV}$ disease risk and $87.8 \%$ with high $\mathrm{CV}$ disease risk did not achieve the LDL-c goal of $<1.8 \mathrm{mmol} / \mathrm{L}^{20,21)}$ Collectively, these data indicated suboptimal LDL-c management in very high-risk T2DM patients.

The risk of CV events increases with higher LDL-c levels and advancing age. In a primary prevention cohort, the risk of myocardial infarction and ASCVD events increased for every $1.0 \mathrm{mmol} / \mathrm{L}$ increase in LDL-c in all age groups, with the highest increase noted in individuals aged $\geq 70$ years. ${ }^{22)}$ A recent meta-analysis reported that lowering LDL-c levels by statin therapy resulted in significant reductions in major vascular events, irrespective of age, including those in patients $>75$ years of age. ${ }^{23)}$ Therefore, the ESC/EAS guidelines for dyslipidemia provide the same recommendations for LDL-c control to older individuals with ASCVD as those for younger patients. ${ }^{17)}$ However, little is known about the optimal control of LDL-c in older individuals. Our study results showed that patients with T2DM at very high CV disease risk often did not achieve their LDL-c goal. This poor achievement suggests the need for efforts to improve the management of hypercholesterolemia in older patients with $\mathrm{T} 2 \mathrm{DM}$ with a very high risk of $\mathrm{CV}$ disease.

\section{Factors Associated with Not Achieving the LDL-c Goal in Patients with T2DM at Very High CV Disease Risk}

Previous studies revealed factors associated with not achieving LDL-c goals, including obesity, female sex, high CV disease risk, no (vs. lower dose) statin therapy, lower (vs. higher) statin dose, and non-adherence to statin therapy. ${ }^{16,24)}$ In patients with diabetes, two studies showed that female sex was a predictor for not achiev- ing LDL-c goals. ${ }^{20,25)}$ However, these studies used higher LDL-c target values and did not focus on the older population. Our study identified obesity and high-intensity statin therapy as factors associated with LDL-c goal non-achievement in patients with T2DM at very high $\mathrm{CV}$ disease risk.

Both obesity and high LDL-c levels are risk factors for CV in patients with T2DM. ${ }^{18)}$ Obesity can induce abnormal changes in lipid metabolism, leading to elevated triglyceride, small dense LDL-c, non-HDL-c, and low HDL-c levels. ${ }^{26)}$ In the Liraglutide Effect and Action in Diabetes: Evaluation of Cardiovascular Outcome Results (LEADER) trial that enrolled high-risk patients with T2DM in 32 countries, the prevalence of obesity was $62.1 \%$ (of them, $73.2 \%$ were $\geq 60$ years of age), and obese patients were less likely to reach defined lipid goals. ${ }^{27)}$ Previous studies also revealed that modest weight loss had positive effects on glycemic control, lipid profiles, and other CV risk factors. ${ }^{28-30)}$ Moreover, the ESC/EASD and American Diabetes Association guidelines identified obesity management as an important target for T2DM treatment. ${ }^{18,31)}$ However, the strategy for weight control for older patients with T2DM is complex because older individuals often present with other aging-related health issues such as frailty, impaired functional status, polypharmacy, and polymorbidity. ${ }^{32)}$ Further longitudinal studies are needed to clarify the optimal BMI range for older adults with T2DM and the impact of BMI on their lipid profiles.

Numerous meta-analyses of data from randomized controlled trials have demonstrated that intensive lowering of LDL-c levels with high-intensity statin therapy provides a significant benefit over low-to-moderate intensity statin therapy in terms of preventing non-fatal CV events. ${ }^{33-35)}$ Furthermore, reaching LDL-c goals was more prevalent in patients treated with high-dose statin thera- 
py than in those treated with low-to-moderate-dose statin therapy. ${ }^{24,36)}$ However, in our study, only $22.4 \%$ of all patients with T2DM with very high CV disease risk received high-intensity statin therapy, which can explain the high prevalence of non-achievement of the LDL-c goal of $<1.4 \mathrm{mmol} / \mathrm{L}$ (89.2\%). The difficulty in managing this very high-risk group was also reported in a recent study in South Korea, which showed that high-intensity statins were prescribed to only $9.1 \%$ of patients with very high risk, with $61 \%$ of these patients not achieving the LDL-c goal of $<1.8$ $\mathrm{mmol} / \mathrm{L}{ }^{37)}$ Suboptimal doses of statins were observed in several Asian countries in the DYSIS-II study, ${ }^{38)}$ mainly due to concerns about the side effects of higher statin doses satisfaction with patients' LDL-c levels. ${ }^{39)}$ Our findings highlight the gap between clinical practice and existing guidelines, in that statin utilization remains inadequate, thus contributing to not achieving LDL-C goals.

\section{Non-achievement of the Non-HDL-c Goal in Patients with T2DM with Very High CV Disease Risk}

Non-HDL-c reflects the full spectrum of atherogenic lipoproteins, rather than LDL-c alone. Among statin-treated patients, the association strength with the risk of major $\mathrm{CV}$ events was greater for non-HDL-c than for LDL-c. ${ }^{40)}$ Furthermore, non-HDL-c was a strong predictor of CV disease in patients with diabetes. ${ }^{41)}$ Thus, the ESC/EAS guidelines for dyslipidemia also define secondary goals based on non-HDL-c levels. ${ }^{17)}$ In our study, $87.9 \%$ of patients did not reach the non-HDL-c goal ( $<2.2 \mathrm{mmol} / \mathrm{L})$. Additionally, those participants who did not meet the LDL-c goal were more likely to also not meet the non-HDL-c goal. Our results revealed suboptimal achievements of LDL-c and non-HDL-c goals, as recommended by the ESC/EAS guidelines, in very high-risk patients and should raise physician awareness regarding the need for more aggressive control of dyslipidemia in these patients. This study has several limitations. First, our study was only performed at urban hospitals and included patients who were managed by geriatricians. Thus, the results may not entirely reflect the lipid goal achievement trend in Vietnam or the practices of cardiology physicians. Second, we could not estimate the effects of different types of statin treatment on lipid goal achievement because there were often switches between brand-name and generic statins and different types of statins. Third, we only obtained data on the intensity of statin therapy within one month before enrollment. Fourth, we did not assess the use of medications that may have influenced the achievement rate of the LDL-c goal or adherence to lipid-lowering treatment. Finally, due to the cross-sectional nature of the study design, we could not evaluate the causal relationships between lipid non-achievement and the related factors.
In conclusion, the results of this study showed that the rate of achieving an aggressive LDL-c goal was suboptimal in older patients with T2DM with a very high CV disease risk in Vietnam. Only $10.8 \%$ reached the recommended LDL-c goal $(<1.4 \mathrm{mmol} / \mathrm{L})$. Obesity increased the probability of not achieving the LDL-c goal, whereas high-intensity statin therapy decreased this probability.

\section{SUPPLEMENTARY MATERIALS}

Supplementary materials can be found via https://doi.org/10. 4235/agmr.21.0099.

\section{ACKNOWLEDGEMENTS}

We thank the patients for their participation in our study.

\section{CONFLICT OF INTEREST}

The researchers claim no conflicts of interest.

\section{FUNDING}

None.

\section{AUTHOR CONTRIBUTIONS}

Conceptualization, HTN; Data curation, AHN, TTN, HML; Investigation, HTN, AHN, T.T.N, HML; Methodology, HTN, KPTH, HML; Project administration, HTN, HML; Supervision, HTN, HML; Writing-original draft, HTN, KPTH; Writing-review \& editing, HTN, AHN, TTN, HML.

\section{REFERENCES}

1. Sinclair A, Saeedi P, Kaundal A, Karuranga S, Malanda B, Williams R. Diabetes and global ageing among 65-99-year-old adults: findings from the International Diabetes Federation Diabetes Atlas, 9th edition. Diabetes Res Clin Pract 2020;162: 108078.

2. Yang YC, Lin MH, Wang CS, Lu FH, Wu JS, Cheng HP, et al. Geriatric syndromes and quality of life in older adults with diabetes. Geriatr Gerontol Int 2019; 19:518-24.

3. Worldometer. World report on aging and health: Vietnam population [Internet]. [place unknown]: Worldometer; c2021 [cited 2021 Nov 12]. Available from: https://www.worldometers. info/world-population/vietnam-population/.

4. Hinh ND, Minh HV. Public health in Vietnam: scientific evidence for policy changes and interventions. Glob Health Action 2013;6:20443. 
5. Ngoc NB, Lin ZL, Ahmed W. Diabetes: what challenges lie ahead for Vietnam? Ann Glob Health 2020;86:1.

6. Nguyen QN, Pham ST, Do LD, Nguyen VL, Wall S, Weinehall L, et al. Cardiovascular disease risk factor patterns and their implications for intervention strategies in Vietnam. Int J Hypertens 2012;2012:560397.

7. Vu HT, Nguyen TX, Nguyen HT, Le TA, Nguyen TN, Nguyen AT, et al. Depressive symptoms among elderly diabetic patients in Vietnam. Diabetes Metab Syndr Obes 2018;11:659-65.

8. Nguyen AT, Pham HQ, Nguyen TX, Nguyen TT, Nguyen HT, Nguyen TN, et al. Knowledge, attitude and practice of elderly outpatients with type 2 diabetes mellitus in National Geriatric Hospital, Vietnam. Diabetes Metab Syndr Obes 2020;13:390917.

9. Islam SM, Purnat TD, Phuong NT, Mwingira U, Schacht K, Froschl G. Non-communicable diseases (NCDs) in developing countries: a symposium report. Global Health 2014;10:81.

10. Low Wang CC, Hess CN, Hiatt WR, Goldfine AB. Clinical update: cardiovascular disease in diabetes mellitus: atherosclerotic cardiovascular disease and heart failure in type 2 diabetes mellitus: mechanisms, management, and clinical considerations. Circulation 2016;133:2459-502.

11. American Diabetes Association. 10. Cardiovascular disease and risk management: standards of medical care in diabetes-2020. Diabetes Care 2020;43(Suppl 1):S111-34.

12. Colhoun HM, Betteridge DJ, Durrington PN, Hitman GA, Neil HA, Livingstone SJ, et al. Primary prevention of cardiovascular disease with atorvastatin in type 2 diabetes in the Collaborative Atorvastatin Diabetes Study (CARDS): multicentre randomised placebo-controlled trial. Lancet 2004;364:685-96.

13. Sever PS, Poulter NR, Dahlof B, Wedel H, Collins R, Beevers G, et al. Reduction in cardiovascular events with atorvastatin in 2,532 patients with type 2 diabetes: Anglo-Scandinavian Cardiac Outcomes Trial lipid-lowering arm (ASCOT-LLA). Diabetes Care 2005;28:1151-7.

14. Knopp RH, d'Emden M, Smilde JG, Pocock SJ. Efficacy and safety of atorvastatin in the prevention of cardiovascular end points in subjects with type 2 diabetes: the Atorvastatin Study for Prevention of Coronary Heart Disease Endpoints in non-insulin-dependent diabetes mellitus (ASPEN). Diabetes Care 2006;29:1478-85.

15. Cholesterol Treatment Trialists' (CTT) Collaborators, Kearney PM, Blackwell L, Collins R, Keech A, Simes J, et al. Efficacy of cholesterol-lowering therapy in 18,686 people with diabetes in 14 randomised trials of statins: a meta-analysis. Lancet 2008; 371:117-25.

16. Kim S, Han S, Rane PP, Qian Y, Zhao Z, Suh HS. Achievement of the low-density lipoprotein cholesterol goal among patients with dyslipidemia in South Korea. PLoS One 2020;15:e228472.

17. Mach F, Baigent C, Catapano AL, Koskinas KC, Casula M, Badimon L, et al. 2019 ESC/EAS Guidelines for the management of dyslipidaemias: lipid modification to reduce cardiovascular risk. Eur Heart J 2020;41:111-88.

18. Cosentino F, Grant PJ, Aboyans V, Bailey CJ, Ceriello A, Delgado V, et al. 2019 ESC Guidelines on diabetes, pre-diabetes, and cardiovascular diseases developed in collaboration with the EASD. Eur Heart J 2020;41:255-323.

19. World Health Organization. The Asia-Pacific perspective: redefining obesity and its treatment. Geneva, Switzerland: World Health Organization; 2000.

20. Breuker C, Clement F, Mura T, Macioce V, Castet-Nicolas A, Audurier Y, et al. Non-achievement of LDL-cholesterol targets in patients with diabetes at very-high cardiovascular risk receiving statin treatment: Incidence and risk factors. Int J Cardiol 2018;268:195-9.

21. Yang YS, Yang BR, Kim MS, Hwang Y, Choi SH. Low-density lipoprotein cholesterol goal attainment rates in high-risk patients with cardiovascular diseases and diabetes mellitus in Korea: a retrospective cohort study. Lipids Health Dis 2020;19:5.

22. Mortensen MB, Nordestgaard BG. Elevated LDL cholesterol and increased risk of myocardial infarction and atherosclerotic cardiovascular disease in individuals aged 70-100 years: a contemporary primary prevention cohort. Lancet 2020;396:164452.

23. Cholesterol Treatment Trialists' Collaboration. Efficacy and safety of statin therapy in older people: a meta-analysis of individual participant data from 28 randomised controlled trials. Lancet 2019;393:407-15.

24. Danchin N, Almahmeed W, Al-Rasadi K, Azuri J, Berrah A, Cuneo CA, et al. Achievement of low-density lipoprotein cholesterol goals in 18 countries outside Western Europe: the International ChoLesterol management Practice Study (ICLPS). Eur J Prev Cardiol 2018;25:1087-94.

25. Bayram F, Sonmez A, Haymana C, Sabuncu T, Dizdar OS, Gurkan E, et al. Utilization of statins and LDL-cholesterol target attainment in Turkish patients with type 2 diabetes: a nationwide cross-sectional study (TEMD dyslipidemia study). Lipids Health Dis 2020;19:237.

26. Klop B, Elte JW, Cabezas MC. Dyslipidemia in obesity: mechanisms and potential targets. Nutrients 2013;5:1218-40.

27. Masmiquel L, Leiter LA, Vidal J, Bain S, Petrie J, Franek E, et al. LEADER 5: prevalence and cardiometabolic impact of obesity in cardiovascular high-risk patients with type 2 diabetes mellitus: baseline global data from the LEADER trial. Cardiovasc Diabe- 
tol 2016; 15:29.

28. Zomer E, Gurusamy K, Leach R, Trimmer C, Lobstein T, Morris $\mathrm{S}$, et al. Interventions that cause weight loss and the impact on cardiovascular risk factors: a systematic review and meta-analysis. Obes Rev 2016;17:1001-11.

29. Wing RR, Lang W, Wadden TA, Safford M, Knowler WC, Bertoni AG, et al. Benefits of modest weight loss in improving cardiovascular risk factors in overweight and obese individuals with type 2 diabetes. Diabetes Care 2011;34:1481-6.

30. Pastors JG, Warshaw H, Daly A, Franz M, Kulkarni K. The evidence for the effectiveness of medical nutrition therapy in diabetes management. Diabetes Care 2002;25:608-13.

31. American Diabetes Association. 8. Obesity management for the treatment of type 2 diabetes: standards of medical care in diabetes-2021. Diabetes Care 2021;44(Suppl 1):S100-10.

32. Longo M, Bellastella G, Maiorino MI, Meier JJ, Esposito K, Giugliano D. Diabetes and aging: from treatment goals to pharmacologic therapy. Front Endocrinol (Lausanne) 2019;10:45.

33. Cannon CP, Steinberg BA, Murphy SA, Mega JL, Braunwald E. Meta-analysis of cardiovascular outcomes trials comparing intensive versus moderate statin therapy. J Am Coll Cardiol 2006; 48:438-45.

34. Mills EJ, O’Regan C, Eyawo O, Wu P, Mills F, Berwanger O, et al. Intensive statin therapy compared with moderate dosing for prevention of cardiovascular events: a meta-analysis of $>40000$ patients. Eur Heart J 2011;32:1409-15.

35. Cholesterol Treatment Trialists' (CTT) Collaboration, Baigent C, Blackwell L, Emberson J, Holland LE, Reith C, et al. Efficacy and safety of more intensive lowering of LDL cholesterol: a me- ta-analysis of data from 170,000 participants in 26 randomised trials. Lancet 2010;376:1670-81.

36. Ferrieres J, Rouyer MV, Lautsch D, Ashton V, Ambegaonkar BM, Brudi P, et al. Suboptimal achievement of low-density lipoprotein cholesterol targets in French patients with coronary heart disease: contemporary data from the DYSIS II ACS/CHD study. Arch Cardiovasc Dis 2017;110:167-78.

37. Yang YS, Lee SY, Kim JS, Choi KM, Lee KW, Lee SC, et al. Achievement of LDL-C targets defined by ESC/EAS (2011) guidelines in risk-stratified Korean patients with dyslipidemia receiving lipid-modifying treatments. Endocrinol Metab (Seoul) 2020;35:367-76.

38. Poh KK, Ambegaonkar B, Baxter CA, Brudi P, Buddhari W, Chiang FT, et al. Low-density lipoprotein cholesterol target attainment in patients with stable or acute coronary heart disease in the Asia-Pacific region: results from the Dyslipidemia International Study II. Eur J Prev Cardiol 2018;25:1950-63.

39. Lee ZV, Llanes EJ, Sukmawan R, Thongtang N, Ho HQ, Barter P, et al. Prevalence of plasma lipid disorders with an emphasis on LDL cholesterol in selected countries in the Asia-Pacific region. Lipids Health Dis 2021;20:33.

40. Brunner FJ, Waldeyer C, Ojeda F, Salomaa V, Kee F, Sans S, et al. Application of non-HDL cholesterol for population-based cardiovascular risk stratification: results from the Multinational Cardiovascular Risk Consortium. Lancet 2019;394:2173-83.

41. Lu W, Resnick HE, Jablonski KA, Jones KL, Jain AK, Howard WJ, et al. Non-HDL cholesterol as a predictor of cardiovascular disease in type 2 diabetes: the strong heart study. Diabetes Care 2003;26:16-23. 